\title{
Simulation of the Stabilizing Mechanism of Distal Radioulnar Joint during Pronation and Supination*
}

\author{
Satoshi SHIMAWAKI**, Motohiro EBE ${ }^{* *}$, Masataka NAKABAYASHI ${ }^{* *}$ and \\ Naotaka SAKAI ${ }^{* *}$ \\ **Graduate School of Engineering, Utsunomiya University, \\ 7-1-2 Yoto, Utsunomiya-shi, Tochigi, 321-8585, Japan \\ E-mail: simawaki@cc.utsunomiya-u.ac.jp
}

\begin{abstract}
The objective of this study was to simulate the movements of the bones involved in human forearm rotation. We developed a biomechanical arm model comprising bone, muscle, and ligament components. Computed tomography (CT) scans of a human arm were used to determine the morphology of the humerus, ulna, radius, and hand bones. Magnetic resonance (MR) images were used to create muscle spring models simulating the pronator teres, pronator quadratus, and supinator muscles. Twenty-seven ligaments connecting the bone components were approximated by wire models. We also created several ligament rupture models by eliminating some of the 27 ligaments. Ligaments were removed according to the following four stages: Stage a-I, only dorsal triangular fibrocartilage complex (TFCC); Stage b-I, only palmar TFCC; Stage II, both dorsal and palmar TFCC; Stage III, both dorsal and palmar TFCC plus distal interosseous membrane. In the complete 27-ligament model and in the ligament rupture models, the forearm was rotated to $90^{\circ}$ supination and $85^{\circ}$ pronation for comparison. In supination, the rupture of the palmar TFCC (Stage b-I) caused a larger difference between the two types of models than the rupture of the dorsal TFCC (Stage a-I). The distal radioulnar joint instability and radial laxity occurred for Stage III rather than Stage II. The distal radioulnar joint was more stable and radial laxity was less pronounced during pronation in the presence of the pronator quadratus muscle than during supination in the absence of this muscle. These findings were in good accord with previous study results.
\end{abstract}

Key words: Musculoskeletal System, Human Dynamics, Joint, Arm Model, Ligament Rupture, CT and MR Images, Simulation, Distal Radioulnar Joint, TFCC

\section{Introduction}

The elbow joint plays an important role in activities of daily living, sports, and work. Extension and flexion of the arm and forearm rotation (i.e., pronation and supination) are the major motions of the elbow joint ${ }^{(1)}$. Pronation is the act of turning the palm downward, while supination is the act of turning the palm upward. In a previous study ${ }^{(2)}$, the authors reported a biomechanical arm model capable of elbow flexion and extension; this model consisted of three bones, three muscles, and 17 ligaments. We used this model to simulate unstable joint behavior associated with ligament rupture and demonstrated the utility of digital human modeling. In this study, we expand the arm model and create a new model

${ }^{*}$ Received 12 May, 2013 (No. 13-00063) [DOI: 10.1299/jbse.8.278]

Copyright $@ 2013$ by JSME 
capable of forearm rotation.

Forearm rotation mainly involves two bones (the radius and ulna). When the elbow joint is in the neutral unrotated position (i.e., at $90^{\circ}$ of elbow flexion with the thumb pointing upward), the two bones are nearly parallel to each other. As the forearm rotates, the radius rolls around the ulna. Thus, forearm rotation necessitates articulation of two joints, namely, the proximal and distal radioulnar joints. In the proximal radioulnar joint, the radial head articulates with the radial notch of the ulna, and this articulation is held together by the annular ligament of the radius and the quadrate ligament of Dènucé. The radius rotates back and forth along the ulna, with the perfect congruency between the radial head and the radial notch of the ulna being supported by the annular ligament. In the distal radioulnar joint, the ulnar notch of the radius meets with the cylindrical head of the ulna, and the two are held together by the distal radioulnar ligaments. The distal radius rotates around the ulnar head during pronation and supination. Therefore, the distal radius crosses the ulna during pronation.

The pronator teres muscle, pronator quadratus muscle, and radial flexor muscle primarily serve to pronate the forearm ${ }^{(3)(4)}$. In this study, we focused on the pronator teres and quadratus muscles to simulate pronation from the neutral unrotated position to the maximum angle. The key muscles that supinate the forearm include the biceps brachii muscle and supinator muscle ${ }^{(3)(4)}$. Here, we focused on the supinator muscle to simulate supination movements with the elbow extended.

Baseball, tennis, and other sports are known to cause injuries to the triangular fibrocartilage complex (TFCC) which is a supporting tissue in the forearm. The TFCC is a group of ligaments that absorb impact between the forearm and wrist bones. The TFCC is composed of the triangular fibrocartilage located at the ulnar side of the wrist and surrounding ligaments ${ }^{(5)}$. Specifically, the TFCC includes the dorsal distal radioulnar ligament, palmar distal radioulnar ligament, palmar ulnolunate ligament, anterior stylopisiform bundle, posterior stylotriquetral bundle, and other components. TFCC injuries lead to unstable forearm rotation and decreased grip strength. Clinically, injuries to the interosseous membrane of the forearm seldom occur in isolation ${ }^{(1)}$; it is often accompanied with injury to the TFCC and annular ligament of the radius ${ }^{(1)(6)}$. These observations suggest that ligament ruptures proceed in a step-wise manner.

The objective of this study was to simulate the movements of the bones involved in human forearm rotation. We developed a biomechanical arm model comprising bone, muscle, and ligament components. Computed tomography (CT) scans of a human arm were used to determine the morphology of the humerus, ulna, radius, and hand bones. Magnetic resonance (MR) images were used to create muscle spring models simulating the pronator teres, pronator quadratus, and supinator muscles. Twenty-seven ligaments connecting the bone components were approximated by wire models. The accuracy of the constructed virtual model was evaluated by comparing the simulation results with MR images of the human arm at different angles of forearm rotation. We also created several ligament rupture models that reflected a step-wise progression of ligament tear. These ligament rupture models were used to analyze the kinematic behavior of the ulna and radius resulting from joint injury.

\section{Methods}

\subsection{Creation of Arm Model}

We created an arm model composed of three components: bones, muscle springs, and ligament wires. These components were developed based on CT and MR imaging scans of the arm of a 22-year-old man (height, $180 \mathrm{~cm}$; weight, $67 \mathrm{~kg}$ ) who had no medical history of injuries or other problems of the right wrist and arm. This study was approved by Ethical Review Committee on Research Involving Human Subject of Utsunomiya University prior 
to its commencement.

\subsubsection{Bone Model}

Multislice CT images (1 mm slice thickness) were taken of the right arm section distal to the head of the humerus with the elbow extended and the neutral unrotated position (Somatom Definition, Siemens). The plane of the slice was perpendicular to the long axis of the bone. The resulting CT images were processed using an image-extraction software (Surf Driver 4.0, David Moody and Scott Lozanoff), and the margins of cortical bone and muscle tissue were visually determined. The margins identified from the CT images were stacked along the long bone axis to construct a bone model ${ }^{(7)}$. The bone model was composed of the humerus, radius, ulna, and hand bones, including the carpal bones (Fig. 1). As each of the small bones constituting the hand does not specifically influence forearm rotation, they were treated as a single functional unit.

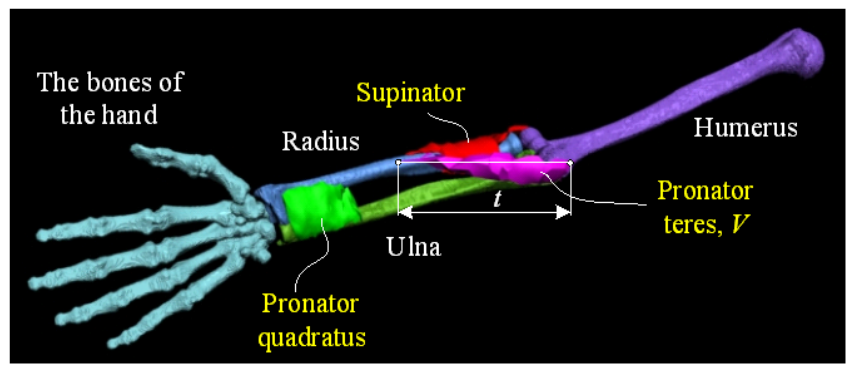

Fig. 1 Bone and muscle models. $t$ : muscle length, $V$ : muscle volume.

\subsubsection{Muscle Spring Model}

First, the three-dimensional geometries of the muscles were digitized from MR images (muscle models). Then, the muscle models were used to determine the points of action and muscle tensions, and the data were used to construct a muscle spring model.

The muscle models were generated as below. Multislice MR images were taken of the right arm distal to the humeral head with the elbow extended and in the neutral unrotated position (3-mm slice thickness, MAGNETOM Avanto A Tim System, Siemens). The plane of the slice was perpendicular to the long axis of the bone. The pronator teres and quadratus muscles were selected as the key muscles regulating forearm pronation, and the supinator muscle was selected as the primary muscle regulating forearm supination ${ }^{(8)(9)}$. These muscles are firmly attached to the bone at both ends. The proximal and distal points of attachment are termed an origin and an insertion, respectively. In a manner similar to the delineation of the bone margin, the image-extraction software was used to visually determine the margins of the muscles, and the images were stacked along the long axis to create models for each muscle (Fig. 1). Anatomical reference images and information were used to determine the accurate geometries of individual muscles ${ }^{(10)}$. The pronator teres muscle mediates forearm pronation. This muscle arises from two points of origin. One is broadly located proximal to the medial epicondyle of the humerus, and the other is a narrow point located inside the coronoid process of the ulna. In this study, the former (i.e., the humeral site) was used as the origin. This muscle has a narrow point of insertion in the middle of the lateral surface of the radius. The pronator quadratus muscle also regulates forearm pronation. This muscle originates from a broad area in the most distal quarter of the anterior ulna, and ends in a broad area in the most distal quarter of the anterior radius. The supinator muscle is involved in forearm supination. This muscle starts from the lateral epicondyle of the humerus, radial collateral ligament, radial annular ligament, and supinator crest of the ulna. In this study, the lateral epicondyle of the humerus was selected as the origin for simulation purposes. The point of insertion is located at the proximal lateral edge 


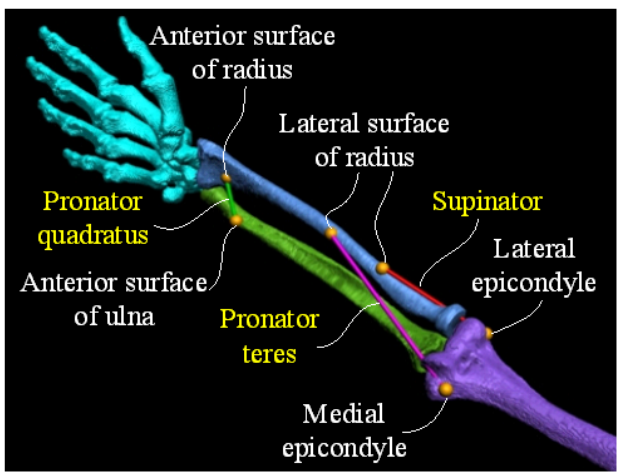

Fig. 2 Muscle spring models generated from muscle models

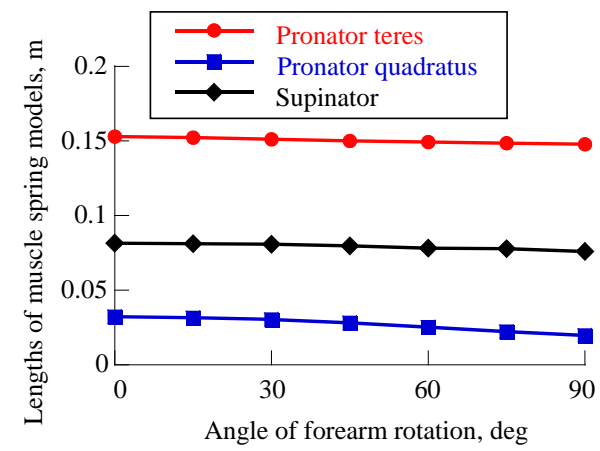

Fig. 3 Relationship between the angle of forearm rotation and the lengths of muscle spring models of the radius.

Next, the muscle spring model was created as described below. Muscle contraction draws the origin and insertion of the muscle closer. This mechanism enables the radius to roll around an axis of rotation. Therefore, the mechanism of muscle contraction was reasonably approximated by a biomechanical model in which a fixed spring with a spring constant was attached to the bone components at the points of origin and insertion. For muscles with a narrow point of origin or insertion, the point was defined as the point of application of force in the spring model. In this study, the point of insertion for the pronator teres muscle and the point of origin for the supinator muscle were determined in this manner. Other sites of origin and insertion were broad. To overcome this problem, we determined the surface areas of overlap between the muscle and bone models, and the center of gravity of the overlapping area was defined as the point of application of spring force. A muscle spring model created in this manner is schematically illustrated in Fig. 2.

The spring constant was selected based on the maximum tension of each simulated muscle. The contraction force is proportional to the length of the hypothetical muscle spring; when the length of the muscle spring is equal to zero, so is the contraction force. Figure 3 graphically illustrates the relations between the muscle spring length and the angle of forearm rotation. The muscle spring lengths slightly decrease with the increase in the angle of forearm rotation, but they are almost constant. The contraction force of a muscle is known to be proportional to its physiological cross-sectional area, which is the sum of the cross-sectional areas of the muscle fibers. The physiological cross-sectional area can be determined on the basis of muscle volume, fiber length, and pennation angle, as shown below. The muscle models constructed from MR scans (Fig. 1) were used to calculate the volume $V$ and length $t$ of the muscle. The ratios of muscle length to muscle fiber length given in the literatures for each muscle ${ }^{(11) \sim(13)}$ were applied to the calculated muscle lengths $t$ to estimate muscle fiber lengths $t^{\prime}$. The pennation angles $\theta$ for the muscles were taken from the literature ${ }^{(4)}$. The physiological cross-sectional area was determined by equation (1) ${ }^{(14)}$ : 


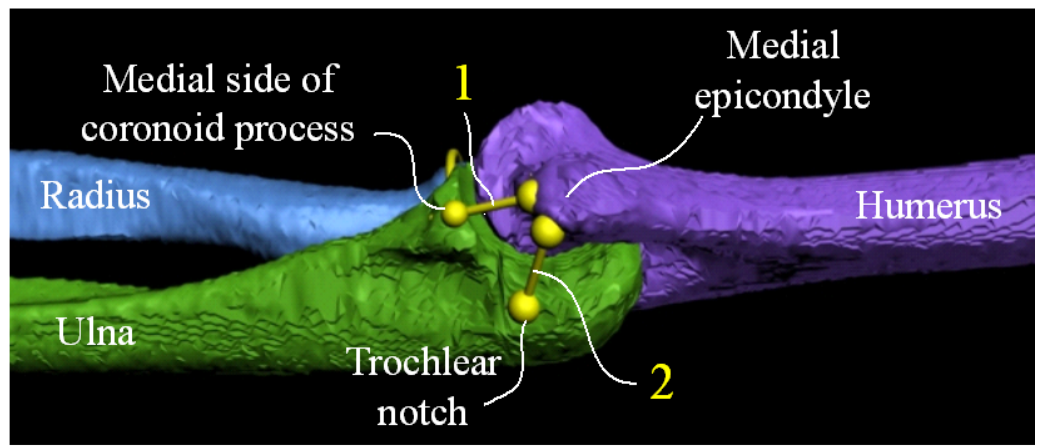

(a) Ulnar collateral ligament. 1:anterior oblique ligament, 2:posterior oblique ligament.

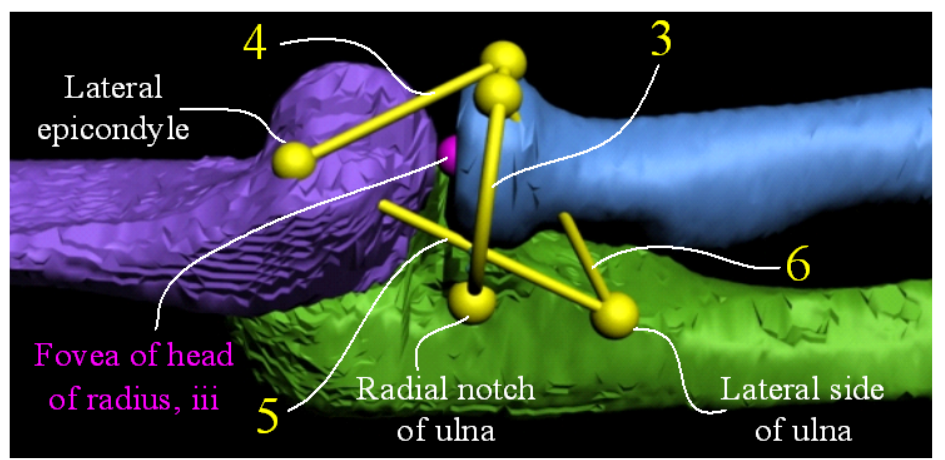

(b) Lateral collateral ligament complex. 3:anular ligament of radius; 4:Radial collateral ligament, 5:lateral ulnar collateral ligament; 6 :accessory posterior annular ligament.

Fig. 4 Ligament wire models on the elbow joint

$$
\text { Physiological cross - sectional area }=\left(\frac{V}{t^{\prime}}\right) \cos \theta
$$

The maximum muscle force per unit physiological cross-sectional area of $48 \mathrm{~N} / \mathrm{cm}^{2}$ was taken from the literature ${ }^{(15)}$. Consequently, the maximum forces of 189,75 , and $150 \mathrm{~N}$ were determined for the pronator teres, pronator quadratus, and supinator muscles, respectively. Next, the spring constants for the pronator teres and quadratus muscles were determined so as to render the contraction force ratio of these muscles at $85^{\circ}$ pronation substantially equal to the ratio of the maximum muscle force values. The spring constant for the supinator muscle was set at a value below the maximum force to allow for a slow rotation.

\subsubsection{Ligament Wire Model}

Ligaments connect bones to other bones and help to stabilize joints. Ligaments restrict the range of motion, thereby playing an important role in motor function. In this study, ligaments were approximated by wire model attached to the bone components (Figs. 4, 5, 6). The ligaments involved in forearm rotation can be roughly classified into the ligaments of the elbow joint, interosseous membrane of the forearm, distal radioulnar joint ligaments, and carpal ligaments.

The ligaments of the elbow joint can generally be divided into the medial collateral ligament and lateral collateral ligament complex ${ }^{(1)(16)(17)}$. The medial collateral ligament is located at the medial aspect of the elbow, and unites the distal aspect of the humerus and the proximal aspect of the ulna. This consists of the anterior oblique (Fig. 4a, 1), posterior oblique (Fig. 4a, 2), and transverse ligaments. The anterior oblique ligament arises from the lower front part of the medial epicondyle of the humerus and inserts onto the medial coronoid process of the ulna. The posterior oblique ligament originates from the lower part 


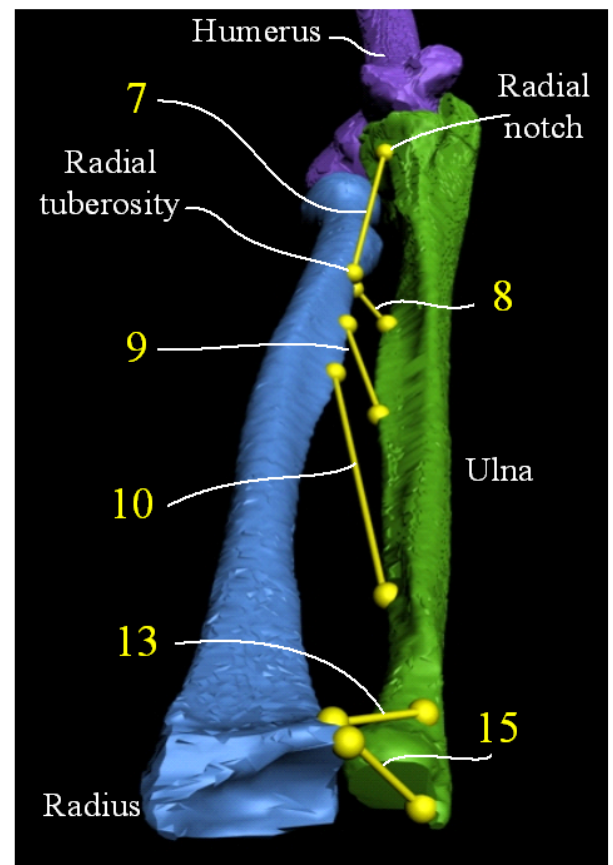

(a) Ligaments of forearm (anterior view). 7:oblique cord; 8:proximal bundle; 9:Middle descending bundle; 10:Distal descending bundle; 13:anterior ligament; 15:palmar radioulnar ligament.

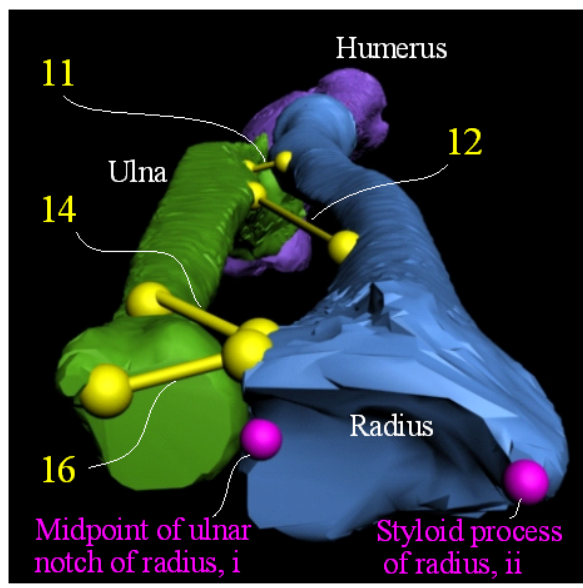

(b) Ligaments of forearm (posterior view). 11:proximal ascending bundle; 12:Distal ascending bundle; 14:posterior ligament; 16: dorsal radioulnar ligament.

Fig. 5 Ligament wire models on the forearm

of the medial epicondyle of the humerus and has a fan-shaped insertion onto the trochlear notch of the ulna. The transverse ligament is attached to the medial aspect of the trochlear notch of the ulna. As this ligament arises from and inserts on the ulna, its contribution to elbow joint stability is minimal ${ }^{(18)}$. Consequently, this ligament was excluded from our model. The lateral collateral ligament complex is composed of the annular ligament of the radius, Dénucé's quadrate ligament, radial collateral ligament (proper), lateral ulnar collateral ligament, and accessory posterior annular ligament. The annular ligament of the radius (Fig. 4b, 3) embraces the radial head, restricting the movement of the radial head. The origin and insertion of the annular ligament occur on the anterior and posterior surface of the radial notch of the ulna, respectively. Dénucé's quadrate ligament (not shown in Fig. $4 \mathrm{~b}$ ) is found between both ends of the annular ligament of the radius, attached to the inferior border of the radial notch of the ulna and to the neck of the radius. The radial collateral ligament (Fig. 4b, 4) originates from the lateral epicondyle of the humerus and terminates on the annular ligament of the radius, and gradually increases in width. The lateral ulnar collateral ligament (Fig 4b, 5), indistinguishable from the lateral collateral ligament at its 


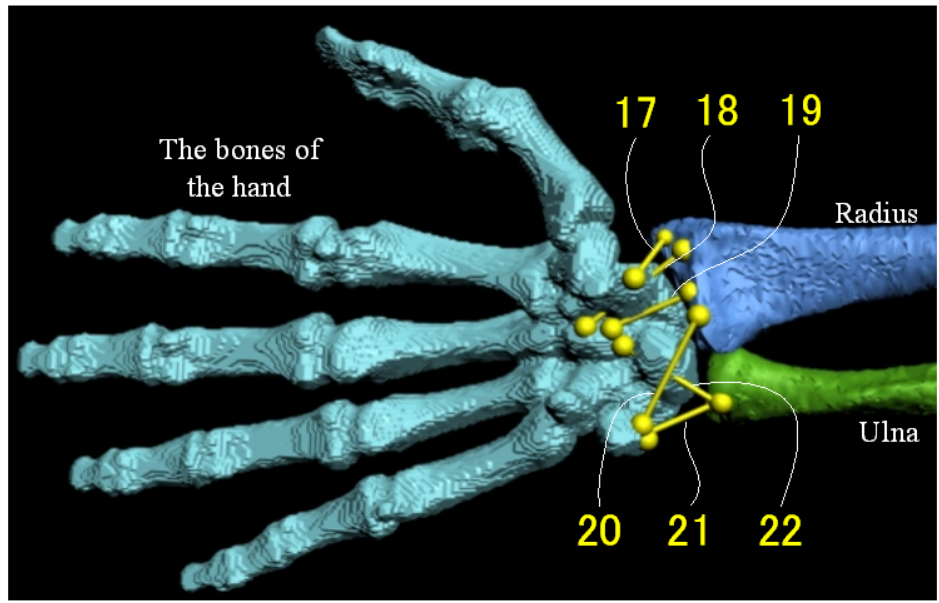

(a) Ligaments of wrist (anterior view). 17:anterior band of radial carpal collateral ligament; 18:radio-capitate ligament, 19 :anterior radio-lunate bund; 20 :anterior radio-triquetral bund; 21:anterior stylo-pisform band; 22:anterior ulno-lunate ligament.

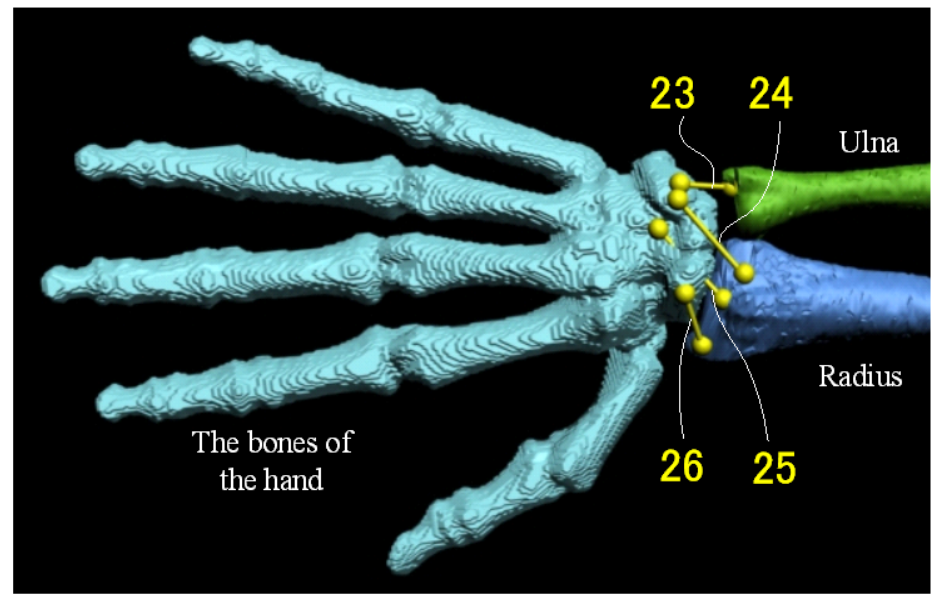

(b) Ligaments of wrist (posterior view). 23: posterior stylo-triquetral band; 24:posterior radio-triquetral bund; 25:posterior radio-lunate bund; 26:posterior band of radial carpal collateral ligament.

Fig. 6 Ligament wire models on the wrist

attachment to the lateral epicondyle, extends to the supinator crest of the ulna. The accessory posterior annular ligament (Fig. 4b, 6) connects to the inferior border of the annular ligament of the radius and to the supinator crest of the ulna.

The interosseous membrane of the forearm can be divided into the oblique cord (Fig. $5 a, 7)$ and two groups of bundles. The anterior group includes the proximal bundle (Fig. 5a, 8), middle descending bundle (Fig. 5a, 9), and distal descending bundle (Fig. 5a, 10), while the posterior group includes the proximal ascending bundle (Fig. 5b, 11) and distal ascending bundle (Fig. 5b, 12). The oblique cord connects the ulna and the radius, and originates from the radial notch of the ulna and inserts below the radial tuberosity. The anterior and posterior groups of bundles confine the proximal and distal movement of the radius, respectively.

The distal radioulnar joint ligaments include the anterior ligament (Fig. 5a, 13), posterior ligament (Fig. 5b, 14), palmar radioulnar ligament (Fig. 5a, 15), and dorsal radioulnar ligament (Fig. 5b, 16). The anterior and posterior ligaments connect the anterior and posterior aspects, respectively, of the distal ends of the radius and ulna. The palmar and dorsal radioulnar ligaments are thin membranes extending between the corresponding surfaces on the palmar and dorsal aspect of the distal radioulnar articulation, respectively. These ligaments enable a stable forearm rotation by limiting radial movement.

The carpal ligaments are classified into palmar and dorsal ligaments ${ }^{(1)}$. The lateral 


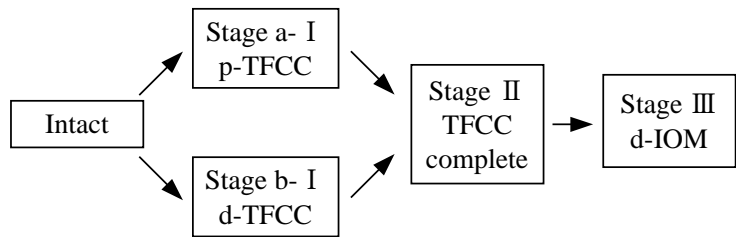

Fig. 7 Order of sectioning in arm model. p-TFCC:palmar TFCC, d-TFCC:dorsal TFCC, d-IOM:distal interosseous membrane.

collateral ligament consists of palmar and dorsal bundles, and the medial collateral ligament is composed of the anterior stylopisiform bundle and posterior stylotriquetral bundle. The palmar ligaments include the anterior bundle of the radial carpal collateral ligament (Fig. 6a, 17), radio-capitate ligament (Fig. 6a, 18), anterior radio-lunate bundle (Fig. 6a, 19), anterior radio-triquetral bundle (Fig. 6a, 20), anterior stylo-pisiform bundle (Fig. 6a, 21), and anterior ulno-lunate ligament (Fig. 6a, 22). The dorsal ligaments include the posterior stylo-triquetral bundle (Fig. 6b, 23), posterior radio-triquetral bundle (Fig. 6b, 24), posterior radio-lunate bundle (Fig. 6b, 25), and posterior band of the radial carpal collateral ligament (Fig. 6b, 26). These ligaments connect carpal bones to the radius and ulna and contribute to the reinforcement of the distal radioulnar ligaments by connecting the radius and ulna indirectly.

On the basis of the consideration presented above, we included the following 27 ligaments in the model: the anterior oblique ligament, posterior oblique ligament, annular ligament of the radius, Dénucé's quadrate ligament, radial collateral ligament, lateral ulnar collateral ligament, accessory posterior annular ligament, oblique cord, proximal bundle, middle descending bundle, distal descending bundle, proximal ascending bundle, distal ascending bundle, anterior ligament, posterior ligament, palmar radioulnar ligament, dorsal radioulnar ligament, anterior radiotriquetral bundle, posterior radiolunate bundle, radiocapitate ligament, dorsal bundle of the lateral collateral ligament, anterior ulnolunate ligament, anterior stylopisiform bundle, posterior stylotriquetral bundle, posterior radiotriquetral bundle, posterior radiolunate bundle, and posterior band of the radial carpal collateral ligament. The ligament origins and insertions were determined based on literature (1)(18)(19)

\subsection{Simulation Conditions}

\subsubsection{Creation of an Arm Model}

An arm model integrating the bone, muscle spring, and ligament wire models was created using a three-dimensional modeling and animation software (3ds MAX 9, Autodesk). The spring contraction force was set at values below the maximum muscle tension. In the initial state, the elbow was extended and the forearm rotation was neutral. The humerus was fixed in a virtual space. For pronation, the supinator muscle was removed from the model, and the springs simulating the pronator teres and quadratus muscles were contracted from the initial state. For supination, the pronator teres and quadratus muscles were removed from the model, and the spring simulating the supinator muscle was contracted from the initial state.

\subsubsection{Ligament Rupture Model}

In order to investigate the effects of ligament rupture on forearm rotation, ligament rupture models were created by eliminating specific ligaments from the complete ligament model. As explained in the Introduction, the TFCC and interosseous membrane of the forearm play a role in stabilizing the forearm joints. In addition, injuries to the interosseous membrane of the forearm do not usually occur in isolation; they are commonly accompanied with TFCC damage. These observations suggest a sequential progression of 


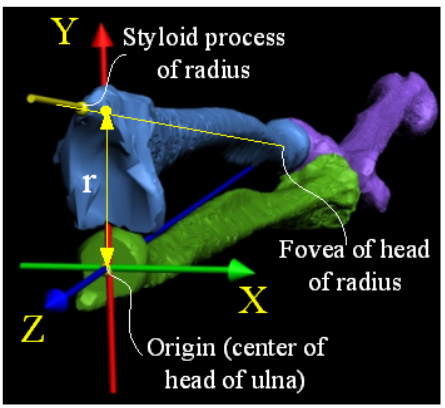

Fig. 8 A local coordinate system. r:the radius of the forearm rotation.

ligament injury.

Therefore, in order to investigate the effects of ligaments on forearm rotation, we removed (ruptured) a part of the ligaments in a stepwise manner from the complete ligament model which has all the ligaments and investigated the stability of forearm rotation of each ligament rupture model. We defined a sequence for removing the ligament wires (a pattern for the progression of ligament rupture affecting forearm rotation), considering the above-mentioned ligament rupture.

As shown in Fig. 7, the respective stages of ligament rupture were as follows: Stage a-I involved ruptures of the dorsal TFCC, i.e., the dorsal radioulnar ligament (Fig. 5b, 16) and the posterior stylotriquetral bundle (Fig. 6b, 23). Stage b-I related to ruptures of the palmar TFCC, i.e., the palmar radioulnar ligament (Fig. 5a, 15), anterior stylopisiform bundle (Fig. 6a, 21), and anterior ulnolunate ligament (Fig. 6a, 22). Stage II included ruptures of both the palmar and dorsal TFCC (a combination of Stage a-I and Stage b-I injuries). Stage III involved, in addition to the Stage II injuries, the ruptures of the distal interosseous membrane complex, i.e., the middle descending bundle (Fig. 5a, 9), distal descending bundle (Fig. 5a, 10), distal ascending bundle (Fig. 5b, 12), anterior ligament (Fig. 5a, 13), and posterior ligament (Fig. 5a, 14).

\subsection{Evaluation Methods}

\subsubsection{Evaluation of the Complete Ligament Model}

In order to evaluate the validity of the complete ligament model, we obtained 3-mm multislice MR images of the subject's forearm rotated at the following angles: neutral $\left(0^{\circ}\right)$; $30^{\circ}, 60^{\circ}$, and $90^{\circ}$ of supination; and $30^{\circ}, 60^{\circ}$, and $90^{\circ}$ of pronation. During image acquisition, the elbow joint was completely stretched, and the forearm rotation angles were achieved by using a fixation device. The upper arm was securely pressed to the chest to prevent shoulder joint rotation. Similar to the creation of the bone models, the resulting scans were used to construct three-dimensional images of the humerus, radius, and ulna at different forearm rotation angles. These images provided data on the locations of the bones for each forearm rotation angle. Geometric differences between these images and the simulation models were calculated to determine simulation accuracy.

In order to determine the relative geometric differences between the measured and simulated data, landmark points of the radius were used for evaluation. Specifically, midpoint of the ulnar notch (Fig. 5b, i) and styloid process (Fig. 5b, ii) were selected for distal sections, and fovea of the radial head (Fig. 4, iii) as proximal section.

In order to calculate the positional differences and rotational angles, a local Cartesian coordinate system for locating the locations and orientations of the ulna and radius was established (Fig. 8). The origin was set at the center of the ulnar head surface. The Z-axis was defined as the direction from the fovea of the radial head to the center of the ulnar head (axis of rotation). The Y-axis was defined as perpendicular to the Z-axis and intersecting the line connecting the fovea of the radial head and the styloid process (radioulnar direction). 


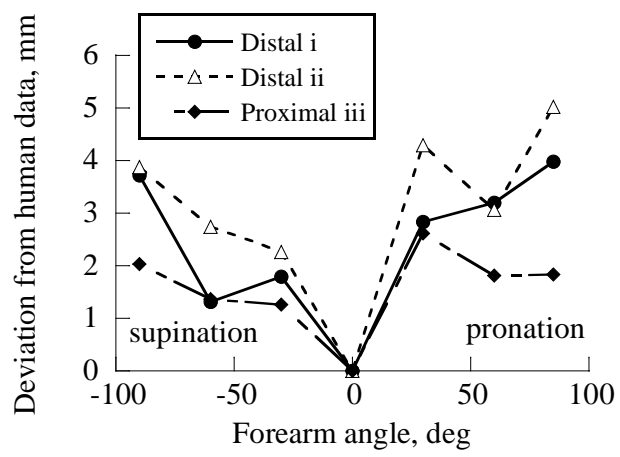

Fig. 9 Comparison of the simulation results with MR images of the human arm during forearm rotation. Distal i: midpoint of ulnar notch of radius; Distal ii: styloid process of radius; Proximal iii: fovea of head of radius.

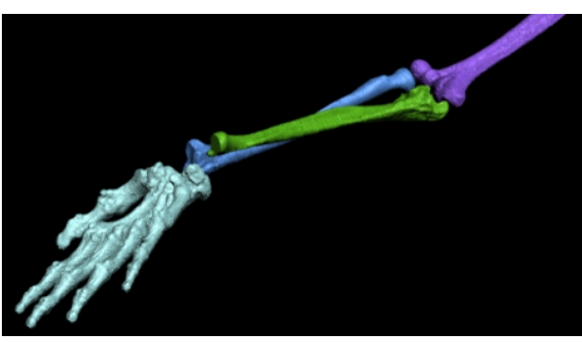

(a) $90^{\circ}$ supination at Stage III

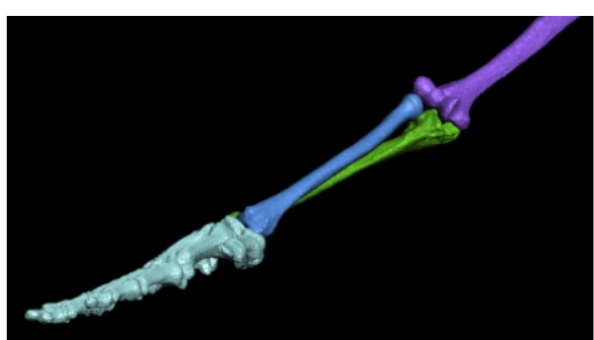

(b) $85^{\circ}$ pronation at Stage III

Fig. 10 Simulation results of the forearm rotation.

The $\mathrm{X}$-axis was defined as a direction perpendicular to $\mathrm{X}$ - and $\mathrm{Y}$-axes and pointing from the dorsal to the palmar side (dorsal-palmar direction). The angles of forearm rotation were determined relative to the line connecting the center of the ulnar head and the radial styloid process at $0^{\circ}$ of rotation. The coordinates of the respective landmark points were determined relative to the coordinate system at the predefined angles of forearm rotation.

\subsubsection{Evaluation of the Ligament Rupture Model}

In order to evaluate the effects of ligament rupture, we focused on the radius of rotation and the position of center of rotation as follows. The radius of rotation was defined as the distance between the center of the ulnar head and a point defined by the intersection of plane $P$ and line $L$. Plane $P$ included the center of the ulnar head and was perpendicular to the line connecting the fovea of the radial head and the center of the ulnar head. Line $L$ passed through the fovea of the radial head and the radial styloid process (the radius of rotation is designated as $r$ in Fig. 8). The center of rotation was defined by the intersection of the XY-plane of the local coordinate system and a line connecting the fovea of the radial head and the center of the ulnar head.

\section{Results}

\subsection{Differences between MR-Based Measurement and Simulation of Forearm Rotation}

The differences between the MR-based measurements and simulation results were determined at different forearm rotation angles, and the results are graphically represented relative to the MR-based measurements in Figure 9. In supination, the largest difference of $3.9 \mathrm{~mm}$ was observed at $90^{\circ}$ for the radial styloid process (ii). In pronation, the largest difference of $5.0 \mathrm{~mm}$ was observed at $85^{\circ}$ for the radial styloid process (ii). The differences were generally greater for the distal sections than for the proximal section.

\subsection{Behavior of the Joints in the Ligament Rupture Model}


Table 1 Deviation from simulation result with intact ligaments (mm)

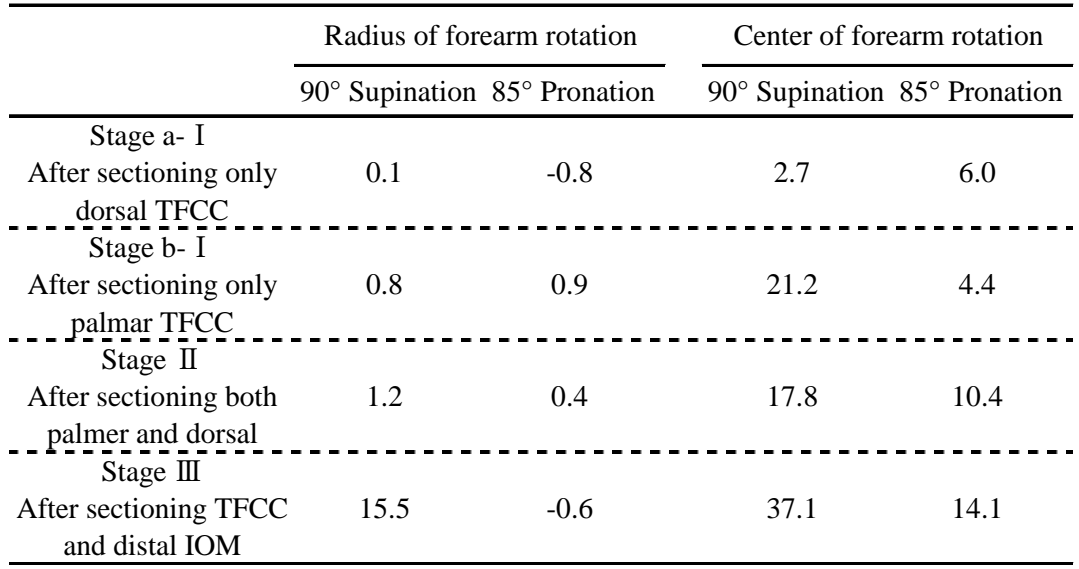

Using the ligament rupture model, we simulated the behaviors of the joints under stage-III ligament rupture at $90^{\circ}$ supination and $85^{\circ}$ pronation, and the results are schematically illustrated in Figure 10 . The distal radioulnar joint was dislocated at $90^{\circ}$ supination (Fig. 10a). In contrast, no dislocation was observed for the distal radioulnar joint at $85^{\circ}$ pronation, indicating a relatively smooth rotation (Fig. 10b). The observed difference can be explained by the presence of the pronator quadratus muscle, which constrained the laxity of the distal radioulnar joint in pronation.

\subsection{Effects of Ligament Rupture Patterns on the Radius and the Position of Center of Rotation}

The differences in the radius and the position of center of rotation between the complete 27-ligament model and the ligament rupture models were determined at $90^{\circ}$ supination and $85^{\circ}$ pronation, and the results are summarized in Table 1 for the respective stages of ligament rupture pattern. No major differences were noted either in the radius of rotation or in the position of center of rotation in Stage a-I injuries (dorsal TFCC rupture only). In Stage b-I (palmar TFCC rupture only), a large difference in the position of center of rotation was observed for supination, although no significant differences in the radius of rotation were noted for either supination or pronation. These results indicate that in supination, the palmar TFCC without the dorsal one leads to small differences from the complete ligament model than does the dorsal TFCC without the palmar one. In Stage II (ruptures of the dorsal and palmar TFCC), the results were relatively similar to those obtained in Stage b-I in supination. In pronation, a moderate increase was noted in the center of rotation. In Stage III (ruptures of the dorsal and palmar TFCCs and distal interosseous membrane), the differences during supination were larger than those observed in Stage II, while the differences in the position of center of rotation during pronation showed only a mild increase from those in Stage II.

These results show that the palmar TFCC helps to stabilize the distal radioulnar joint in supination. These results also indicate that the pronator quadratus muscle and the distal interosseous membrane prevent widening of the radioulnar distance. Our findings indicate that forearm rotation will be secured as long as the pronator quadratus muscle and the distal interosseous membrane function properly, even if the dorsal and palmar TFCC are disrupted.

\section{Discussion}

The maximum difference between the MR-based measurements and computer-based simulations was no more than about $5 \mathrm{~mm}$. Similar degrees of difference were reported in our previous work ${ }^{(2)}$. Considering that the MR slice thickness was $3 \mathrm{~mm}$, the differences 
were probably attributable to the errors produced in constructing the simulation models. The differences were small for the fovea of the radial head (iii) (proximal), as this part was located close to the axis of rotation. In contrast, the radial styloid process (ii) (distal) was located at a farther distance from the ulna, and had a greater range of motion, which might lead to the increased difference. These differences may account for the differences observed between the radial styloid process (ii) and the fovea of the radial head (iii). However, no inadequate component behaviors, such as dislocation or insufficient range of rotation, were noted in the complete ligament model. Thus, this arm model which enables simulation of forearm rotation can be applied to the evaluation of ligament functions in forearm rotation.

Palmer classified TFCC injuries into traumatic lesions (Class 1) and degenerative lesions (Class 2) ${ }^{(20)}$. He further divided traumatic lesions into four subgroups by location. Class 1A lesion relates to a tear or perforation of the horizontal portion of the TFCC and is therefore unrelated to distal radioulnar joint instability. Class $1 \mathrm{~B}$ lesions represent traumatic avulsions of the TFCC from its insertion into the distal ulna. This subgroup and the subsequent ones involve distal radioulnar joint instability. Class 1C lesions represent avulsions of the TFCC from its distal attachment to the lunate or triquetrum. Class 1D lesions represent traumatic avulsions of the TFCC from its attachment to the radius, including injuries to the distal radioulnar ligament. Among the ligament tear patterns investigated in the present study, Stage II was related to Palmer Class 1B to 1D. Melone and Nathan described TFCC injuries as a continuum of injury beginning at the ulnar styloid and classified them into five basic stages of increasing severity ${ }^{(21)}$. Stage 1 relates to a detachment of the articular disk from its insertion at the base of the ulnar styloid. Stage 2 represents an avulsion of the extensor carpi ulnaris subsheath. Stage 3 represents a disruption of the ulnocarpal (i.e., ulnolunate and ulnotriquetral) ligaments. Stage 4 relates to the rupture of the lunotriquetral interosseous ligament, whereas Stage 5 relates to a disruption of the midcarpal ligament. Stage II injury patterns examined in this study corresponded to Stages 1-3 of the injuries described by Melone and Nathan.

In the present study, the distal palmar and dorsal radioulnar ligaments were simulated by using a single wire, respectively. Our simulation showed that the distal palmar radioulnar ligament becomes taut at maximum supination to sustain the stability of the distal radioulnar joint. This finding agrees with the results of several studies using cadaver wrist specimens ${ }^{(22) \sim(24)}$. This biomechanical simulation study provided new evidence supporting the results of in vitro studies concerning the role of distal radioulnar ligaments in stabilizing the distal radioulnar joint. Although controversial, several authors proposed to divide the distal portion of the palmar and dorsal radioulnar ligaments into superficial and deep segments and to assign them different roles in rotation (25)(26). More specifically, the superficial and deep fibers of the palmar radioulnar ligament become taut and relaxed, respectively, at maximum supination, whereas the superficial and deep fibers of the dorsal radioulnar ligament become relaxed and taut, respectively, at maximum pronation. In this study, this deep fiber structure was not introduced and the above-mentioned phenomena could not be investigated. If the deep fiber structure was considered in our arm model, it might function as a backup for the joint stability and provide more stable results.

A comparison of Stage II and Stage III results shows that distal radioulnar joint instability and radial laxity were more pronounced after distal interosseous membrane ruptures (Table 1 and Fig. 10). In supination, the radius and the position of center of rotation in Stage III was $14 \mathrm{~mm}$ and $20 \mathrm{~mm}$ larger than those in Stage II, respectively. This observation indicates that the distal interosseous membrane plays a significant role in stabilizing the distal radioulnar joint. Hotchkiss et al. showed that the interosseous membrane functions to stabilize the distal radioulnar joint by constraining the radius ${ }^{(6)}$. Kihara et al. demonstrated that dissection of both the distal palmar and dorsal radioulnar ligaments did not compromise distal radioulnar joint stability when the interosseous 
membrane was intact ${ }^{(27)}$. Watanabe et al. showed that the distal interosseous membrane constrained laxity of the radius at the distal radioulnar joint ${ }^{(28)}$. Our study supports these previous findings.

Our simulation showed that the distal radioulnar joint was more stable and radial laxity was less pronounced during pronation in the presence of the pronator quadratus muscle than during supination in the absence of this muscle (Table 1 and Fig. 10). At Stage III, the radius and the position of center of rotation during supination was $16 \mathrm{~mm}$ and $23 \mathrm{~mm}$ larger than those during pronation, respectively. This finding indicates that the pronator quadratus muscle is also important in stabilizing the distal radioulnar joint. In a study using fresh cadaver upper extremities, Kihara et al. serially cut the dorsal and palmar radioulnar ligaments, pronator quadratus muscle, and interosseous membrane, and measured the radial displacements generated by the procedure ${ }^{(27)}$. Specifically, the ablations proceeded in the following order: (i) dorsal radioulnar ligament or palmar radioulnar ligament, (ii) remaining radioulnar ligament, (iii) distal interosseous membrane and pronator quadratus muscle, and (iv) remaining part of the interosseous membrane. The results showed that whereas disruption of the dorsal and palmar radioulnar ligaments did not significantly impact the stability of the distal radioulnar joint, disruption of all four components (i.e., dorsal radioulnar ligament, palmar radioulnar ligament, pronator quadratus muscle, and interosseous membrane) caused instability of the joint. Similarly, King et al. demonstrated that the pronator quadratus muscle served to stabilize the distal radioulnar joint ${ }^{(29)}$. These results are in good accord with those of the present study.

We showed that the TFCC, distal interosseous membrane, and pronator quadratus muscle contribute to the stabilization of the distal radioulnar joint. It has been reported that the extensor carpi ulnaris tendon sheath ${ }^{(30)}$ and the distal radioulnar joint capsule ${ }^{(31)}$ also function as stabilizers. In future, adding these factors to the present model will enable clinical conditions to be simulated more precisely.

\section{Conclusion}

In this study, we developed an arm model comprising bone, muscle, and ligament components. CT scans of a human arm were used to determine the morphology of the humerus, ulna, radius, and hand bones. MR images were used to create muscle spring models simulating the pronator teres, pronator quadratus, and supinator muscles. Twenty-seven ligaments connecting the bone components were approximated by wire models. The accuracy of the constructed virtual simulation model was evaluated by comparing the results with MR images of the human arm at different angles of forearm rotation. Then, the simulation model was used to analyze bone kinematics at different levels of ligament rupture. Consequently, the following conclusions were obtained:

(1) The largest differences between MR-based measurements and arm model simulation were $3.9 \mathrm{~mm}$ in supination and $5.0 \mathrm{~mm}$ in pronation, and both were observed for the distal styloid process of the radius. The MR slice thickness (3 $\mathrm{mm})$ may have significantly contributed to these differences. The complete ligament model showed no dislocation or other abnormal behaviors.

(2) The ligament rupture model showed that in supination, the palmar TFCC without the dorsal one (Stage a-I) leads to small differences from the complete ligament model than does the dorsal TFCC without the palmar one (Stage b-I). This observation supports previous in vitro studies demonstrating that the distal palmar radioulnar ligament became taut at maximum supination to sustain the stability of the distal radioulnar joint.

(3) In the ligament rupture model,the distal radioulnar joint instability and radial laxity were more pronounced after distal interosseous membrane ruptures (Stage III) than ruptures of dorsal and palmar TFCC (Stage II). 
(4) The distal radioulnar joint was more stable and radial laxity was smaller during pronation in the presence of the pronator quadratus muscle than during supination in the absence of the muscle.

\section{References}

(1) Kapahdji, A.I., The Physiology of the Joints. Volume 1:The upper limb, Churchill Livingstone, 6th edition (2007), pp.76-145.

(2) Shimawaki, S. and Sakai, N., Simulation of Elbow Flexion with a Three-dimensional Arm Model, Transactions of JSME, Series C, Vol.78, No.788 (2012), pp.1180-1191.

(3) Ikeda, K., Function Anatomy of the Upper Extremity, Bulletin of the Japanese Society of Prosthetic and Orthotic Education, Research and Development, Vol.15, No.3 (1999), pp.204-212.

(4) Holzbaur, K.R., Murray, W.M. and Delp, S.L., A Model of the Upper Extremity for Simulating Musculoskeletal Surgery and Analyzing Neuromuscular Control, Ann. Biomed. Eng., Vol.33, No.6 (2005), pp.829-840.

(5) Palmer, A.K. and Werner, F.W., The Triangular Fibrocartilage Complex of the Wrist Anatomy and Function, J. Hand Surg., Vol.6A, No.2 (1981), pp.153-162.

(6) Hotchkiss, R.N, An, K.N., Sowa, D.T. and Weiand, A.J, An Anatomic and Mechanical study of the Interosseous membrane of the Forearm: Pathomechanics of Proximal Migration of the Radius, J. Hand Surg., Vol.14A, No.2 (1989), pp.256-261.

(7) Shimawaki, S. and Sakai, N., Quasi-static Deformation Analysis of a Human Finger Using a Three-dimensional Finite Element Model Constructed from CT Images, Journal of Environment and Engineering, Vol. 2, No.1(2007), pp.56-63.

(8) Wolf-Heidegger, G., Atlas of Human Anatomy 1 Systemic Anatomy, Body Wall, Upper and Lower Limbs, Medical Sciences International, (2002), pp.82-167.

(9) Schünke, M., Schulte, E., Schumacher, U., Voll, M. and Wesker, K., PROMETHEUS:Atlas of Anatomy General Anatomy and Musculoskeletal System (in japanese), Igaku Shoin, (2008), pp.236-347.

(10) Manaster, B.J., Andrews, C.L., Petersilge, C.A., Crim, J., Grossman, J.W., Roberts, C.C., Miller, T.T. and Rosenberg, Z.S., Managing editor, Sanders, R.K., Diagnostic and Surgical Imaging Anatomy, Musculoskeletal, Amirsys, (2006), pp.95-123.

(11) Kawakami, Y., Nakazawa, K., Fujimoto, T., Nozaki, D., Miyashita, M. and Fukunaga, T., Specific Tension of Elbow Flexor and Extensor Muscles Based on Magnetic Resonance Imaging, Eur. J. Appl. Physiol. Occup. Physiol., Vol.68, No.2 (1994), pp139-147.

(12) Doyle, J.R. and Botte, M.J., Surgical Anatomy of the Hand and Upper Extremity, Lippincott Williams \& Wilkins, (2002), p107.

(13) Holzbaur, K.R., Murray, W.M., Gold, G.E. and Delp, S.L., Upper Limb Muscle Volumes in Adult Subjects, J. Biomech., Vol.40, No.4 (2007), pp.742-749.

(14) Saito, A., Skeletal Muscle Structure, Rigakuryoho Kagaku, Vol.18, No.1 (2002), pp.49-53.

(15) Fukunaga, T., Absolute Muscle Strength of Human - Analysis of Muscle Strength and Body Composition Using Ultrasonic Sensor (in japanese), Kyorin Shoin, (1978), pp.106-131.

(16) Regan, W. D., Korinek, S. L., Morrey, B., F. and An, K-N., Biomechanical Study of Ligaments Around the Elbow Joint, Clin. Orthop. Relat. Res., No.271 (1991), pp170-179.

(17) Morrey, B. F., and An, K-N., Articular and Ligamentous Contributions to the Stability of the Elbow Joint, Am. J. Sports Med., Vol.11, No.5 (1983), pp315-319.

(18) Kobayashi, A., Futami, T. and Moriguchi, T., Function Anatomy of the Medial Collateral Ligament of the Elbow, Clinical orthopedic surgery, Vol.41, No.12 (2006), pp.1247-1250.

(19) Goto, A., Moritomo, H., Murase, T., Oka, K., Sugamoto, K., Arimura, T., Nakajima, Y., Yamazaki, T., Sato, Y., Tamura, S., Yoshikawa, H. and Ochi, T., In Vivo Elbow Biomechanical Analysis During Flexion: Three-dimensional Motion Analysis Using 
Magnetic Resonance Imaging, J. Shoulder Elbow Surg., Vol.13, No.4 (2004), pp.441-447.

(20) Palmer, A.K., Triangular Fibrocartilage Complex Lesions: A Classification, J. Hand Surg., Vol.14A, No.4 (1989), pp.594-606.

(21) Melone, C.P. and Nathan, R., Traumatic Disruption of the Triangular Fibrocartilage Complex, Clin. Orthop. Relat. Res., No.275 (1992), pp.65-73.

(22) Schuind, F., An, K.-N., Gerglund, L., Rey, R., Cooney III, W.P., Linscheid, R.L. and Chao, E.Y.S., The Distal Radioulnar Ligaments:A Biomechanical Study, J. Hand Surg., Vol.16A, No.6 (1991), pp.1106-1114.

(23) Acosta, R., Hnat, W. and Scheker, L.R., Distal Radio-ulnar Ligament Motion During Supination and Pronation, J. Hand Surg., Vol.18B, No.4 (1993), pp.502-505.

(24) Ward, L.D., Ambrose, C.G., Masson, M.V. and Levaro, F., The Role of the Distal Radioulnar Ligaments, Interosseous Membrane, and Joint Capsule in Distal Radioulnar Joint Stability, J. Hand Surg., Vol.25A, No.2 (2000), pp.341-351.

(25) Hagert, C.G., Distal Radius Fracture and the Distal Radioulnar Joint - Anatomical Considerations, Handchir. Mikrochir. Plast. Chir., Vol.26, No.1 (1994), pp.22-26.

(26) $\mathrm{Xu}$, J. and Tang, J.B., In vivo Changes in Lengths of the Ligaments Stabilizing the Distal Radioulnar Joint, J. Hand Surg., Vol.34A, No.1 (2009), pp.40-45.

(27) Kihara, H., Short, W.H., Werner, F.W., Fortino, M.D. and Palmer, A.K., The Stabilizing Mechanism of the Distal Radioulnar Joint During Pronation and Supination, J. Hand Surg., Vol.20A, No.6 (1995), pp.930-936.

(28) Watanabe, H., Berger, R.A., Berglund, L.J., Zobitz, M.E. and An, K.N., Contribution of the Iterosseous Membrane to Distal Radioulnar Joint Constraint, J. Hand Surg., Vol.30A, No.6 (2005), pp.1164-1171.

(29) King, G.J., McMurtry, R.Y., Rubenstein, J.D. and Ogston, N.G., Computerized Tomography of the Distal Radioulnar Joint: Correlation with Ligamentous Pathology in a Cadaveric Model, J. Hand Surg., Vol.11A, No.5 (1986), pp.711-717.

(30) Spinner, M. and Kaplan, E.B., Extensor Carpi Ulnaris. Its Relationship to the Stability of the Distal Radio-ulnar Joint, Clin. Orthop. Relat. Res., Vol.68, (1970), pp.124-129.

(31) Watanabe, H., Berger, R.A., An, K.-N., Berglund, L.J. and Zobitz, M.E., Stability of the Distal Radioulnar joint Contributed by the Joint Capsule, J. Hand Surg., Vol.29A, No.6 (2004), pp.11614-1120. 